\title{
Clinical utility of FDG PET in Parkinson's disease and atypical parkinsonism associated with dementia
}

\author{
Zuzana Walker ${ }^{1,2}$ (1) - Federica Gandolfo ${ }^{3}$. Stefania Orini ${ }^{3}$. Valentina Garibotto ${ }^{4}$ - Federica Agosta ${ }^{5}$. Javier Arbizu ${ }^{6}$. \\ Femke Bouwman ${ }^{7}$. Alexander Drzezga ${ }^{8} \cdot$ Peter Nestor $^{9,10}$ - Marina Boccardi ${ }^{11,12}$. Daniele Altomare ${ }^{12,13}$. \\ Cristina Festari ${ }^{12,13} \cdot$ Flavio Nobili $^{14} \cdot$ for the EANM-EAN Task Force for the recommendation of FDG PET for Dementing \\ Neurodegenerative Disorders
}

Received: 11 April 2018 / Accepted: 16 April 2018 / Published online: 19 May 2018

(C) The Author(s) 2018

\begin{abstract}
Purpose There are no comprehensive guidelines for the use of FDG PET in the following three clinical scenarios: (1) diagnostic work-up of patients with idiopathic Parkinson's disease (PD) at risk of future cognitive decline, (2) discriminating idiopathic PD from progressive supranuclear palsy, and (3) identifying the underlying neuropathology in corticobasal syndrome.

Methods We therefore performed three literature searches and evaluated the selected studies for quality of design, risk of bias, inconsistency, imprecision, indirectness and effect size. Critical outcomes were the sensitivity, specificity, accuracy, positive/ negative predictive value, area under the receiving operating characteristic curve, and positive/negative likelihood ratio of FDG PET in detecting the target condition. Using the Delphi method, a panel of seven experts voted for or against the use of FDG PET based on published evidence and expert opinion.

Results Of 91 studies selected from the three literature searches, only four included an adequate quantitative assessment of the performance of FDG PET. The majority of studies lacked robust methodology due to lack of critical outcomes, inadequate gold standard and no head-to-head comparison with an appropriate reference standard. The panel recommended the use of FDG PET for all three clinical scenarios based on nonquantitative evidence of clinical utility.

Conclusion Despite widespread use of FDG PET in clinical practice and extensive research, there is still very limited good quality evidence for the use of FDG PET. However, in the opinion of the majority of the panellists, FDG PET is a clinically useful imaging biomarker for idiopathic PD and atypical parkinsonism associated with dementia.
\end{abstract}

Zuzana Walker

z.walker@ucl.ac.uk

$\triangle$ Flavio Nobili

flaviomariano.nobili@hsanmartino.it

1 Division of Psychiatry, University College London, London, UK

2 St Margaret's Hospital, Essex Partnership University NHS Foundation Trust, Epping CM16 6TN, UK

3 Alzheimer Operative Unit, IRCCS S. Giovanni di Dio, Fatebenefratelli, Brescia, Italy

4 Division of Nuclear Medicine and Molecular Imaging, Department of Medical Imaging, University Hospitals of Geneva, Geneva University, Geneva, Switzerland

5 Neuroimaging Research Unit, Institute of Experimental Neurology, Division of Neuroscience, San Raffaele Scientific Institute, Vita-Salute San Raffaele University, Milan, Italy

6 Department of Nuclear Medicine, Clinica Universidad de Navarra, University of Navarra, Pamplona, Spain
7 Department of Neurology \& Alzheimer Center, Amsterdam Neuroscience, VU University Medical Center, Amsterdam, The Netherlands

8 Department of Nuclear Medicine, University Hospital of Cologne, University of Cologne and German Center for Neurodegenerative Diseases (DZNE), Cologne, Germany

9 German Center for Neurodegenerative Diseases (DZNE), Magdeburg, Germany

10 Queensland Brain Institute, University of Queensland and the Mater Hospital, Brisbane, Australia

11 LANVIE (Laboratoire de Neuroimagerie du Vieillissement), Department of Psychiatry, University of Geneva, Geneva, Switzerland

12 LANE - Laboratory of Alzheimer's Neuroimaging \& Epidemiology, IRCCS S. Giovanni di Dio, Fatebenefratelli, Brescia, Italy

13 Department of Molecular and Translational Medicine, University of Brescia, Brescia, Italy

14 Department of Neuroscience (DINOGMI), University of Genoa \& Clinical Neurology Polyclinic IRCCS San Martino-IST, Genoa, Italy 
Keywords FDG PET · Parkinson's disease · Progressive supranuclear palsy · Prodromal PD · Corticobasal syndrome · Corticobasal degeneration

\section{Introduction}

There are no clear diagnostic guidelines on the role of FDG PET in the diagnostic work-up of cognitive disorders and dementia. Therefore, the European Association of Nuclear Medicine (EANM) and the European Academy of Neurology (EAN) came together in a joint initiative to provide guidance to clinicians on the use of FDG PET in the context of neurodegenerative diseases. The initiative included a set of 21 clinical scenarios, captured as PICO (Population, Intervention, Comparison, Outcome) questions, that were addressed based on literature evidence and expert consensus [1].

In this article, we focus on the assessment of the quality of studies investigating the clinical utility of FDG PET for identifying patients with Parkinson's disease (PD) who are at risk of cognitive decline, and the utility of FDG PET in facilitating the differential diagnosis of common forms of parkinsonism, i.e. idiopathic PD, including the prodromal stage, progressive supranuclear palsy (PSP) syndromes, and corticobasal syndrome (CBS). Multiple system atrophy (MSA), another neurodegenerative disorder presenting with parkinsonism was not included in this review. MSA very seldom, if ever, affects cognition and therefore it did not fall within our prespecified condition restricting this review to studies on the role of FDGPET in "the diagnostic work-up of cognitive disorders and dementia".

\section{Parkinson's disease}

PD is a common degenerative disorder. It is associated with an increased incidence of cognitive impairment and dementia. The pathology underlying this cognitive decline is variable: while in some patients it is purely Lewy body pathology, in many it is due to mixtures of amyloid, tau and Lewy body pathology [2]. Any therapeutic intervention to stop cognitive decline is likely to be most effective in the early stages of PD or even during prodromal stages of PD. Consequently, it may become important to identify patients at high risk of cognitive decline before its onset. Older age, scores from nonmotor assessments, reduced dopamine transporter uptake in the caudate, deficit on smell testing, CSF amyloid $\beta$ (A $\beta 42$ ) to t-tau ratio, and $\mathrm{APOE} \varepsilon 4$ status are all known risk factors for cognitive decline in patients with newly diagnosed PD [3]. Here we report on the role of FDG PET in predicting cognitive decline in PD.

Not all patients presenting with parkinsonism have idiopathic PD. There are alternative pathologies that can present with parkinsonism and cognitive decline. Both PSP and CBS can mimic PD in early stages and are particularly difficult to diagnose in prodromal stages.

\section{Progressive supranuclear palsy}

The underlying neuropathology of PSP is a characteristic fourrepeat tau neuropathology [4]. There are a number of clinical phenotypes of PSP which consist of different combinations of motor, gait, language, cognitive and behavioural features [5]. The two most common phenotypes are PSP Richardson's syndrome (PSP-RS) and the clinical phenotype that most closely mimics idiopathic PD, PSP-parkinsonism (PSP-P) which often presents with asymmetrical tremor, bradykinesia and rigidity. An initial positive response to levodopa treatment can be misleading. Frequently only later in the disease course, when patients develop additional features of impaired ocular movements with vertical supranuclear gaze palsy, is a retrospective diagnosis of PSP-P made.

\section{Corticobasal syndrome}

CBS is an atypical parkinsonian syndrome which consists of dystonia, rigidity, akinesia, myoclonus, tremor and poor response to levodopa. Typically, there is quite marked asymmetry, including limb apraxia and the alien limb phenomenon. Other features include speech and language impairment and cognitive decline. The term CBS describes a clinical phenotype which has been shown to have a heterogeneous underlying pathology. Corticobasal pathology is found only in about $50 \%$ of all clinically diagnosed patients. This has led to the distinction between the clinical syndrome (CBS) and the pathological diagnosis (corticobasal degeneration, CBD). The other pathologies found at autopsy include Alzheimer's disease (AD), PSP and other tauopathies, dementia with Lewy bodies and Creutzfeldt-Jakob disease. Based on this background, three literature searches were performed to assess the quality of evidence supporting the use of FDG PET in facilitating the diagnosis of PD and atypical parkinsonism associated with dementia where the underlying pathology is PSP or CBD.

\section{Methods}

Seven panellists, four from the EANM and three from the EAN, were appointed to produce recommendations, taking into consideration the incremental value of FDG PET as an add-on investigation to a comprehensive clinical/ neuropsychological assessment, in facilitating the diagnosis 
and management of patients with parkinsonism. Consensus recommendations were reached through a Delphi procedure which was based on the expertise of the panellists. The panellists were provided with comprehensive data regarding the availability and quality of evidence, which was assessed by an independent methodology team, as described by Boccardi et al. [6]. Briefly, we searched the literature using harmonized PICO (Population, Intervention, Comparison, Outcome) questions. Thematic keywords were generated by experts, and studies were selected based on eligibility criteria described elsewhere [6]. Relevant data were extracted from selected studies and assessed for quality of methodology, according to European Federation of Neurological Societies guidance [7] and for their relevance to FDG PET studies [6].

\section{PICO questions for this review}

In this review, the PICO questions asked whether "performing FDG PET would add diagnostic value (in terms of increased accuracy compared with neuropathological diagnosis, biomarker-based diagnosis or diagnosis at follow-up) to standard clinical/neuropsychological assessment alone", to:

- Identify brain dysfunction related to cognitive deterioration in patients with PD and cognitive impairment (PICO question 12)

- Discriminate PSP from PD (PICO question 13)

- Identify the underlying pathological process in patients with CBS (PICO question 15).

\section{Eligibility criteria}

Only original full papers published in English in international journals with an impact factor were considered. Reviews, management guidelines, abstracts and 'grey' literature were excluded. Any sample size was permissible if pathology was the gold standard for diagnosis. Otherwise, the minimum sample size was five for PSP and CBS, and 20 for PD.

\section{Literature search}

Electronic searches were performed using a harmonized keywords string based on the specific PICO question, and included a selection of terms chosen for being largely inclusive to identify a broad range of papers. The strings contained a common part for FDG PET and a PICO question-specific part [6]. The MEDLINE, Embase and Google Scholar databases were searched for studies published up to November 2015. The first screening of all included studies was performed by the panellist responsible for that PICO question (an expert neurologist, psychiatrist or nuclear physician) who could include additional studies based on personal knowledge or forward tracking from the references of papers. The full text of these potentially eligible studies was then independently assessed for eligibility by the methodology team.

\section{Data extraction and quality assessment}

To assess the evidence, data on study features, population of interest, index test, gold/reference standard, and critical/proxy outcomes were extracted (for more details see, Boccardi et al. [6]). Data assessors for this review were D.A. for PICO question 12, and F.G. and S.O. for PICO questions 13 and 15. Critical outcomes were the validated measures of test performance accuracy, sensitivity, specificity, AUC, positive and negative predictive values (PPV, NPV) and likelihood ratios (LR). Additional proxy outcomes for PICO questions 13 and 15 were accuracy of differential diagnosis between typical and atypical parkinsonism. The prediction of years of survival was an additional outcome specific to PICO question 15.

The quality of evidence was consensually assessed by the methodology team based on the study design, gold/reference standard, FDG PET image assessment method (visual or semiquantitative), risk of bias, index test imprecision, applicability, effect size, and effect inconsistency. A final assessment of the relative availability of evidence was formulated, taking into account evidence available from all 21 PICO questions. This ranking was summarized as 'very poor/lacking', 'poor', 'fair' or 'good'. For further details about data extraction and quality assessment see Boccardi et al. [6].

\section{Results}

Among the three PICO questions included in this review, only four of the 91 studies examined included the critical outcomes needed for the three questions of interest (Fig. 1). Our data extraction and assessment failed to find definitive evidence for the clinical utility of FDG PET for identifying neurodegeneration associated with cognitive decline in PD and for discriminating PSP from PD. However, we ranked the evidence supporting the clinical use of FDG PET for discriminating between different underlying pathologies in patients with CBS as "fair" (see Table 1, and Nobili et al. [1]).

\section{PICO question 12: Identify PD-related neurodegeneration associated with impaired cognition}

Of the 111 studies identified and screened by the leading panellist (Z.W.), 21 were sent to the methodology team for data extraction and assessment (see Fig. 1). Eleven studies were excluded for the following reasons: seven [11-17] provided no critical outcomes or did not have the minimum sample size, two $[18,19]$ did not include the target population (i.e. PD 

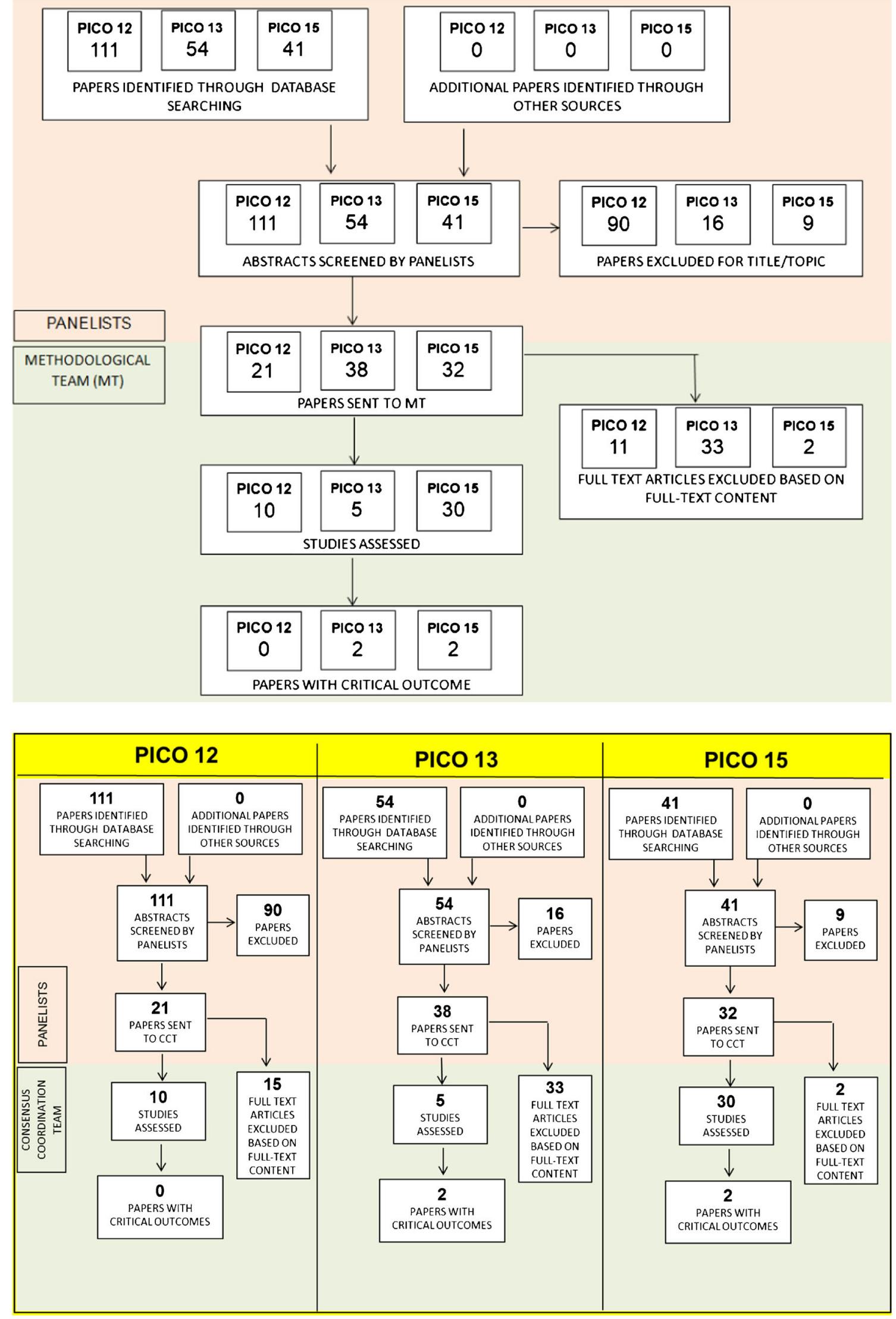

Fig. 1 PRISMA flow chart of selected studies to identify brain dysfunction related to cognitive deterioration (PICO question 12), to discriminate PSP from PD (PICO question 13), and to identify the underlying pathological process in patients with CBS (PICO question 15) [8] 
Table 1 Availability of evidence and panellists' decisions supporting the use of FDG PET in the diagnostic work-up of the main forms of parkinsonism

\begin{tabular}{|c|c|c|c|}
\hline PICO question & $\begin{array}{l}\text { Relative availability } \\
\text { of evidence }\end{array}$ & $\begin{array}{l}\text { Panellists' } \\
\text { recommendations }\end{array}$ & Main reasons for final decision \\
\hline 12 (PD-related decline) & Very low/lacking & Yes & $\begin{array}{l}\text { Sensitive to cortical involvement before } \\
\text { cognitive deficits appear }\end{array}$ \\
\hline 13 (PSP) & Very low/lacking & Yes & Presence of typical hypometabolic patterns \\
\hline $15(\mathrm{CBS})$ & Fair & Yes & Presence of typical hypometabolism \\
\hline
\end{tabular}

Delphi decisions for the other PICO questions of the EANM/EAN initiative led to supporting FDG PET in all clinical scenarios [1] with the exception of preclinical cases [9] and of amyotrophic lateral sclerosis and Huntington's disease [10]

patients without cognitive impairment), in one [20] the index investigation was proton magnetic resonance spectroscopy, and one [21] focused on cognitive impairment and cerebral hypometabolism in PD patients with mild cognitive impairment (PD-MCI) with and without visual hallucinations. The table showing the full data extraction is available at: https://drive. google.com/file/d/0B0_JB3wzTvbpUHRSZ2NfSVZfVkE/ view?usp=sharing.

The critical outcomes were not available in any of the examined studies. The metabolic patterns associated with PDrelated cognitive impairment (i.e. MCI or dementia) as compared with the patterns in patients with no PD-related cognitive impairment were described by Yong et al. [22]. They showed that in comparison with $\mathrm{PD}$ patients with no cognitive impairment, patients with PD dementia (PDD) showed greater metabolic deficits in the parietal and frontal regions but that metabolic patterns in PDD patients and patients with Lewy body dementia were broadly similar.

Two longitudinal studies did identify specific patterns of hypometabolism in PD patients who developed cognitive decline during follow-up compared with the patterns in PD patients who did not develop cognitive impairment. In the first study, 6 of 23 patients with PD developed dementia during follow-up. Patients with progression to PDD had decreased metabolism in the visual association cortex, posterior cingulate cortex and caudate nucleus at baseline (at a time when no major cognitive impairment was present). With progressive impairment, the hypometabolism became more widespred widely to the neocortical regions [23]. In the second study, PD patients who declined had hypometabolism in the posterior and temporal areas of the brain at baseline, in contrast to PD patients with stable cognition over time [24]. However, a quantitative assessment of discrimination accuracy was not provided. Three further studies [25-27] analysed data from the same sample of patients and showed decreased prefrontal and parietal metabolism in PD-MCI patients relative to PD patients with no cognitive impairment, as well as an increase in brainstem/cerebellar metabolism.

The availability of formal evidence supporting the utility of FDG PET for identifying PD-related neurodegeneration in patients with $\mathrm{PD}$ at risk of cognitive impairment was therefore 'lacking'. Nevertheless, the consensus-based recommendation was achieved on Delphi round IV, with five panellists voting for the clinical use of FDG PET based on the presence of cortical hypometabolism in PD patients prior to the onset of cognitive decline (Fig. 2).

\section{PICO question 13: Discriminate PSP from PD}

Of 54 studies identified and screened by the assigned panellist (F.N.), 38 were sent to the methodology team (see Fig. 1), and of these 33 were excluded: 20 studies did not include PSP [28] or PD patients [29-47], 5 studies did not directly and exclusively compare PSP and idiopathic PD [38, 48-51] and the remaining 7 studies [52-58] reported associations only. The data extraction table is available at: https:/drive.google.com/ file/d/0B0_JB3wzTvbpNnEwTmRzX3pmdlU/view?usp= sharing). Critical outcomes were available in two of the examined studies (see Table 2), which included 36 patients

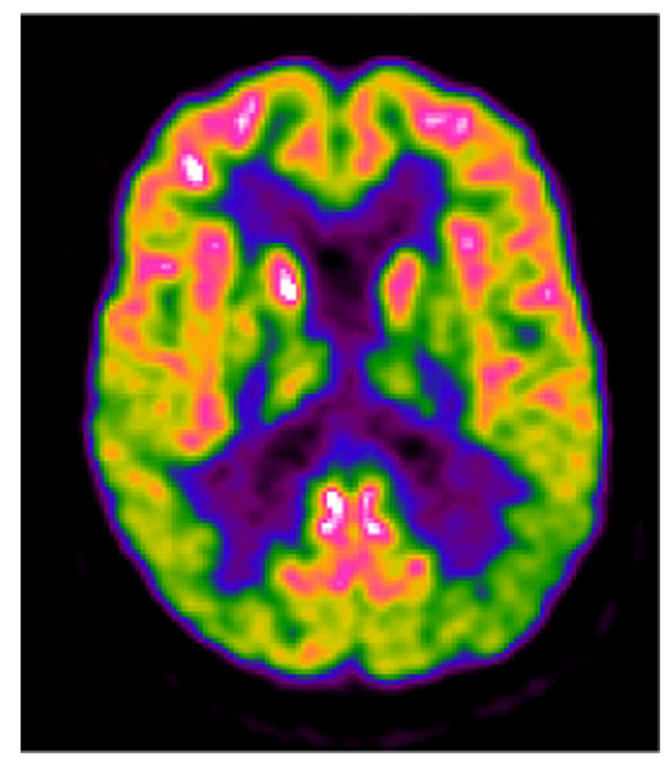

Fig. 2 A 67-year-old patient with de novo PD and subtle cognitive impairment (amnestic MCI), MMSE score 29/30 and high educational level. The FDG PET image shows bilateral posterior parieto-occipital hypometabolism with sparing of the posterior cingulate cortex 


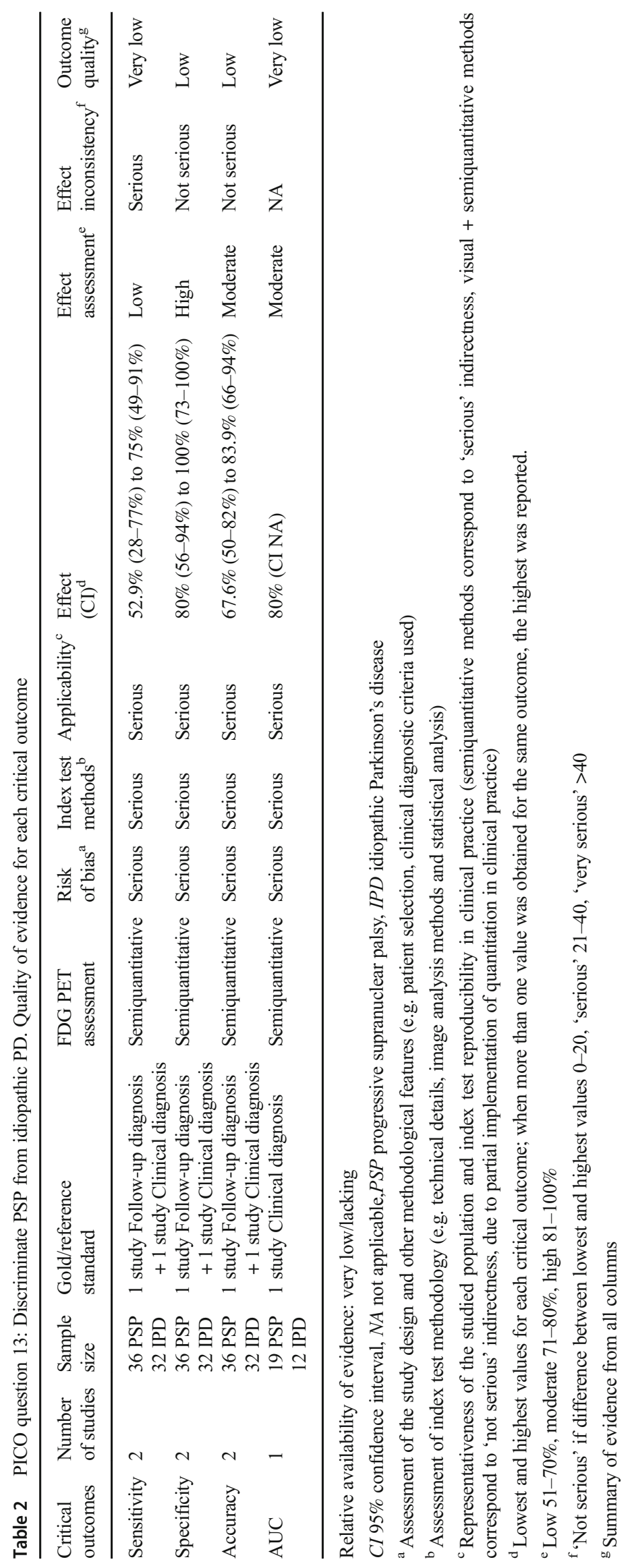


with PSP and 32 with idiopathic PD. In these studies FDG PET was able to discriminate PSP from PD with sensitivities of $52.9-75 \%$, specificities of $80-100 \%$ and accuracies of 67 . 6-83.9\% [59, 60], and an AUC of 0.80 [60].

Additional outcomes were reported in three additional studies [61-63] using a two-step classification, consisting first of discriminating idiopathic PD from atypical parkinsonism, and then discriminating PSP from other atypical parkinsonism. In these studies, FDG PET demonstrated good sensitivity in discriminating idiopathic PD from atypical parkinsonism (range 83-86\%) and a moderate sensitivity in diagnosing PSP and discriminating it from CBD and MSA (range 73-88\%). Specificity and PPV were both greater than $90 \%$ in discriminating idiopathic PD from atypical parkinsonism (range 94\%-97\% and $96 \%-98 \%$, respectively) and in discriminating various subtypes of atypical parkinsonism (range 90\%-94\% and 85\%$96 \%$, respectively). The NPV for discriminating idiopathic PD from atypical parkinsonism was moderate (range 76-83\%).

The five studies had a moderate risk of bias regarding the index test. In the studies by Mudali et al. [59] and Srulijes et al. [60], the level of blindness of the FDG PET reader to the clinical diagnosis was unclear and in those by Teune et al. [57] and again Srulijes et al. [60], the execution of the index test was not described in sufficient detail to allow replication. Further, there was low applicability of all studies due to all studies having included a selected population and having used a semiquantitative method of image analysis.

Taking into account the available evidence for the PICO questions of the entire project, the level of evidence supporting the clinical use of FDG PET for discriminating PSP from idiopathic PD was considered as 'lacking'. However, the consensus recommendation to support the use of FDG PET was defined on Delphi round III and was based on the presence of a typical metabolic pattern of hypometabolism in PSP which predominantly affects the frontal, thalamic, striatal and midbrain regions (Fig. 3).

\section{PICO question 15: Identify the underlying pathological process in patients with corticobasal syndrome}

Among the 41 studies identified by the assigned panellist (F.N.), 32 were sent to the methodological team (see Fig. 1). Two studies $[38,59]$ were excluded because they did not include CBS patients. The table reporting the data extraction is available at: https://drive.google.com/file/d/0B0 JB3wzTvbpamRDZnNpV2VQSE0/view?usp=sharing). Critical outcomes were available in two of the examined studies (Table 3). Both studies [64, 65] used amyloid-PET as the gold standard for clinical diagnosis, and tested the ability of FDG PET to predict AD pathology in a total of 39 patients with CBS. Sensitivity ranged from $91 \%$ to $95 \%$, specificity from $58 \%$ to $75 \%$, and accuracy from $73 \%$ to $82 \%$. Taswell

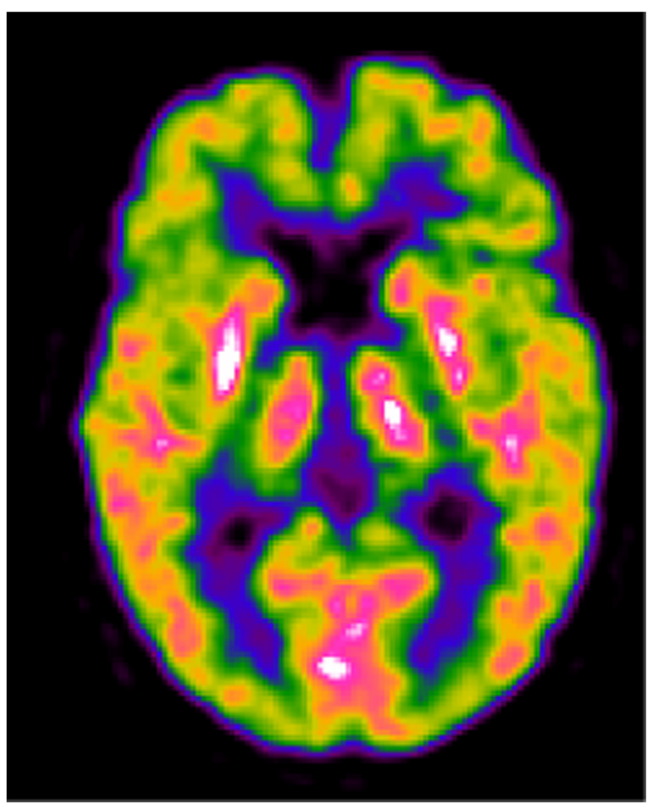

Fig. 3 A 58-year-old patient with PSP and mild cognitive impairment (non-amnesic multiple domain MCI; MMSE score 26/30). The FDG PET image shows moderate hypometabolism of the bilateral frontal cortex, including the medial frontal gyri, and hypometabolism of the caudate nuclei mainly in the right hemisphere, and in the right thalamus

et al. [65] also reported a PPV of 68\%, NPV of 97\%, LR+ of 3. 90 and LR- of 0.06 .

Both studies had a low risk of bias for all items, and moderate applicability concerns. Because of the paucity of results, accuracy of differential diagnosis between CBD and idiopathic PD or other atypical parkinsonism, and prediction of years of survival were added as additional outcomes. They were extracted from 9 of the 28 remaining studies:

- Differential diagnosis between CBS and other atypical parkinsonism was described in four studies [48, 50, 51, 61]. They showed moderate-to-good sensitivity (81$91 \%$ ) and very good specificity (91-100\%).

- Differential diagnosis between CBD and PSP was analysed in four studies [30, 32, 41, 54]. They showed good AUC (0.92-0.97), moderate sensitivity (76-79\%) and heterogeneous specificity (69-92\%).

- Predictability of survival: in the study by Hellwig et al. [54], FDG PET alone was an independent predictor of survival in patients with parkinsonism (age-adjusted hazard ratio: 5.15 for PSP/CBS FDG PET diagnosis).

- Cordato et al. [66] reported similar hypometabolic patterns in autopsy-confirmed CBD and PSP.

In all of these studies, the most frequent pattern of hypometabolism associated with CBS included asymmetrical hypometabolism of the parietal and frontal cortex, thalamus and basal ganglia. The occipital cortex and cerebellum were usually spared. Considering the entire project, the availability of formal evidence supporting the clinical use of FDG PET in 


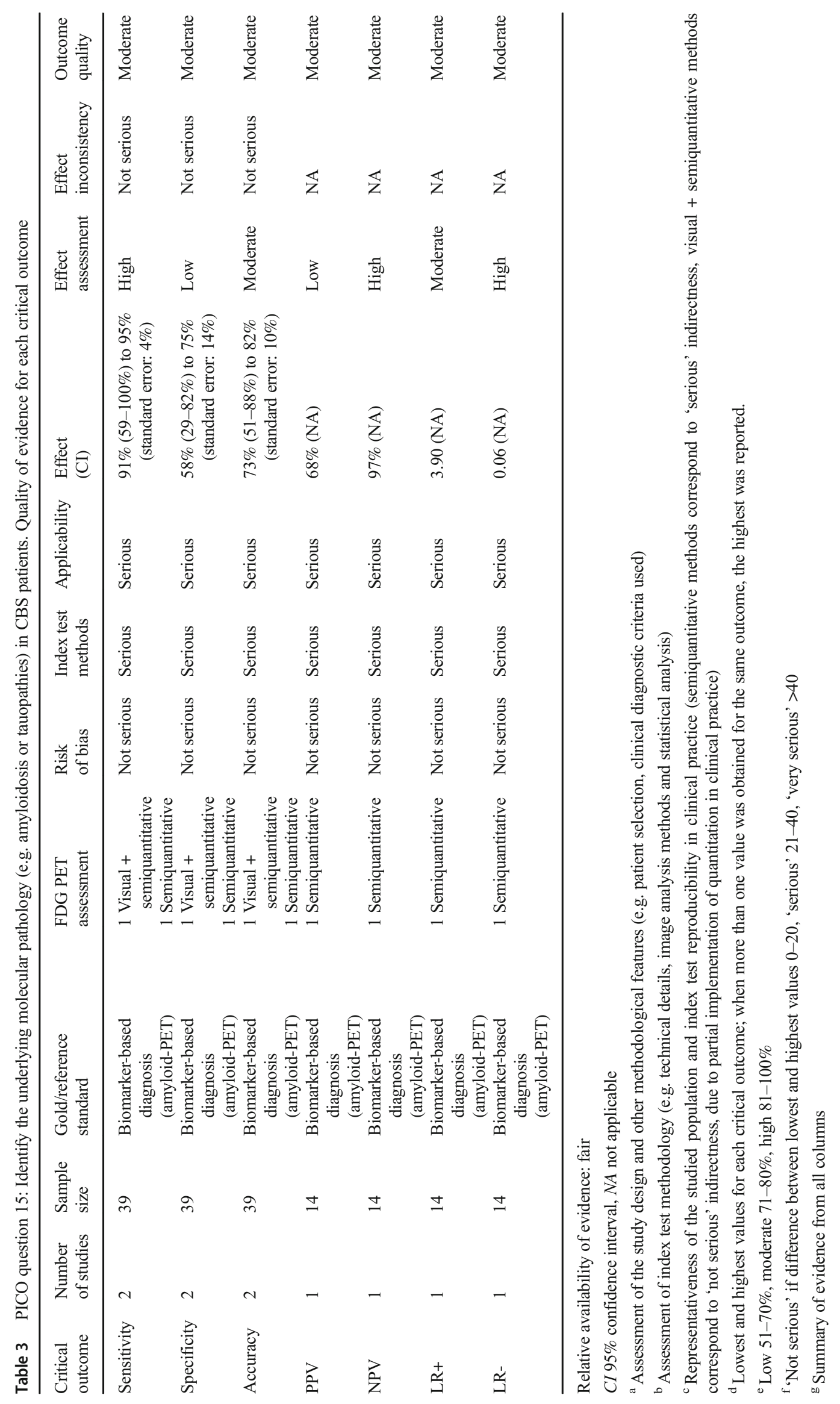


identifying the underlying neuropathology in CBS patients was considered as 'fair'. Consensus was achieved on Delphi round I, with six panellists supporting the clinical use of FDG PET based on the specific asymmetry of the pattern of hypometabolism (Fig. 4).

\section{Discussion}

We assessed the evidence for the clinical utility of FDG PET in diagnosing idiopathic PD and atypical parkinsonism associated with dementia. The evidence supporting the clinical use of FDG PET for identifying PD-related neurodegeneration associated with impaired cognition and for discriminating PSP from idiopathic PD was lacking. Conversely, the evidence supporting the clinical use of FDG PET for identifying the underlying neuropathology in patients with CBS was fair. Despite the low quality of evidence available from published studies, the Delphi panel voted for recommending the clinical use of FDG PET in the differential diagnosis of conditions characterized by parkinsonism and dementia (PICO question 12).

The reasons given by panellists during the Delphi panel process focused on both the clinical utility of the NPV of FDG PET, and also on the PPV of typical patterns of hypometabolism. In particular for the identification of impending PD-related cognitive decline, the panellists recommended the clinical use of FDG PET because patients with PDD or PD-MCI have a typical pattern of

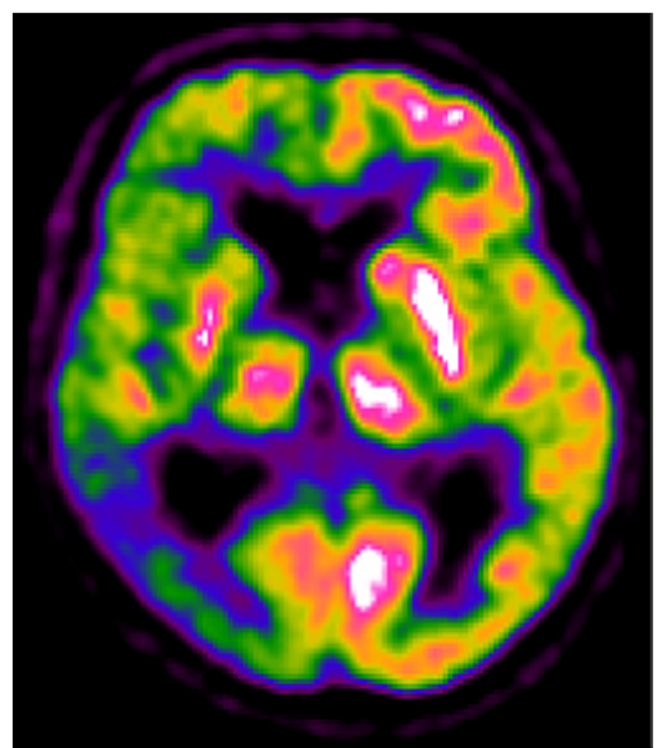

Fig. 4 A 76-year-old patient with CBS and mild cognitive impairment (non-amnestic, multiple domain MCI; MMSE score 28/30). The FDG PET image shows severe hypometabolism in the whole right hemisphere, including the basal ganglia and thalamus, with more severe hypometabolism in the temporoparietal cortex. Dopamine transporter SPECT imaging showed severely reduced uptake in the right basal ganglia, suggesting CBD hypometabolism mainly affecting the posterior cortical areas. The prognostic value of FDG PET was also considered to be clinically relevant in the identification of patients who may benefit from early cholinesterase inhibitor treatment or other future symptomatic treatments. A recently published study [67] showed that FDG PET with statistical parametric mapping detected patterns of hypometabolism that predicted the risk of a patient with PD having progressed to dementia by 4 years with $85 \%$ sensitivity and $88 \%$ specificity. However, this study was not available at the time of the literature search and therefore was not considered in the Delphi process. Atypical hypometabolism of mainly posterior cortical areas on FDG PET could therefore be added to the list of other helpful investigations which include reduced dopamine transporter uptake in the caudate, CSF amyloid $\beta$ (A $\beta 1-42)$ to t-tau ratio and $\mathrm{APOE} \varepsilon 4$ status [3], and is a risk factor for cognitive deterioration in idiopathic PD.

For PICO question 13, panellists based their decision to support the clinical use of FDG PET for discriminating PSP from idiopathic $\mathrm{PD}$ on the presence of a typical metabolic pattern for PSP, which is not present in PD. PSP is characterized by hypometabolism in the medial frontal and anterior cingulate cortices, and in the striatum and midbrain. FDG PET may therefore be useful in early stages of the disease, when the clinical diagnosis is less certain. Perfusion SPECT, albeit less precise because of poorer spatial resolution, also displays a consistent pattern $[68,69]$. The described abnormalities in PSP can be difficult to detect in very early stages by visual analysis alone and a semiautomated assessment, comparing the pattern in the patient with the pattern in age-matched controls is recommended in addition to visual reading, consistent with recommendations of the present EANM-EAN initiative [70]. While a PSPrelated pattern has been repeatedly demonstrated [33, 62, 71], data are still incomplete if different PSP phenotypes are considered, such as PSP-P or pure akinesia with gait freezing. These may be characterized by less severe or incomplete patterns, compared with typical and full-blown PSP. The panellists' decision was consistent with both the EANM procedural guidelines [72] and the more recent diagnostic criteria of the Movement Disorder Society [5, 73], which support the use of FDG PET for discriminating PD from atypical parkinsonian syndromes.

Regarding CBS (PICO question 15), an asymmetrical cortical hypometabolism is known to affect the hemisphere contralateral to the side with akinetic-rigid parkinsonism and apraxia. This hypometabolism is typically found in the motor and premotor cortices, but may also involve the prefrontal or posterior parietal and lateral temporal cortex, and the cingulate gyrus. The heterogeneity of metabolic patterns found in CBS when no autopsy diagnosis is available is most likely due to the variety of different pathologies that can present as CBS. These include $\mathrm{CBD}, \mathrm{PSP}, \mathrm{AD}$ and frontotemporal dementia, and a mix of these conditions. The future challenge is to differentiate the metabolic 
patterns associated with different underlying pathologies and will require either neuropathological confirmation of the diagnosis by autopsy or the use of additional imaging methods, for example tau-PET and amyloid-PET as the gold standard.

Considering the large number of studies published, the fact that the majority had severe limitations and did not allow the generation of evidence that could confidently support the clinical use of FDG PET in the three PICO scenarios was striking. It is important that future studies seek to recruit sufficient numbers of patients and have a robust clinical diagnosis and follow-up and ideally include additional imaging or other biomarkers to strengthen diagnostic certainty. Studies should preferably include autopsy diagnosis as the gold standard. Other important considerations are appropriate clinical comparisons, innovative statistical analysis and the use automatic semiquantitative assessment in addition to visual assessment, to help the nuclear medicine physician interpret findings and to increase specificity and/or sensitivity. In the case of PD, analysing FDG PET using a spatial covariance method could identify characteristic patterns of metabolism in PD with and without cognitive decline. This could be helpful in future research not only to aid more accurate diagnosis but also to evaluate the effect of new therapeutic interventions [74].

Acknowledgments The procedure for assessing scientific evidence and defining consensual recommendations was funded by the European Association of Nuclear Medicine (EANM) and by the European Academy of Neurology (EAN). We thank the Guidelines Working Group of EAN, particularly Simona Arcuti and Maurizio Leone, for methodological advice.

Funding This project was partially funded by the European Association of Nuclear Medicine (EANM) and the European Academy of Neurology (EAN).

\section{Compliance with ethical standards}

Conflicts of interest Zuzana Walker received grants and tracers, personal fees for consultancy and a speaker's fee from G.E. Healthcare.

Federica Gandolfo declares that she has no conflicts of interest.

Stefania Orini declares that she has no conflicts of interest.

Valentina Garibotto declares that she has no conflicts of interest.

Federica Agosta is Section Editor of NeuroImage: Clinical; has received speaker fees from Biogen Idec, Novartis, and Excellence in Medical Education; and receives or has received research support from the Italian Ministry of Health, AriSLA (Fondazione Italiana di Ricerca per la SLA), and the European Research Council. She received personal fees from Elsevier INC.

Javier Arbizu received grants from Eli-Lilly \& Co, Piramal and GE Healthcare.

Femke Bouwman declares that he has no conflicts of interest.

Alexander Drzezga received grants and nonfinancial support from EliLilly \& Co, Siemens and GE Healthcare; he also received nonfinancial support from Piramal.

Peter Nestor declares that he has no conflicts of interest.

Marina Boccardi received funds from the European Association of Nuclear Medicine(EANM) to perform the evidence assessment and the global coordination of the present project. She also received research grants from Piramal and has served as a paid member of advisory boards for Eli Lilly.
Daniele Altomare received a grant allocated by the European Academy of Neurology (EAN) for data extraction and evidence assessment for the present project.

Cristina Festari declares that she has no conflicts of interest.

Flavio Nobili received personal fees and nonfinancial support from GE Healthcare, nonfinancial support from Eli-Lilly and grants from Chiesi Farmaceutici.

Ethical approval This is a review article that does not describe any original studies with human participants performed by any of the authors. Ethical approval is shown in each of the original quoted studies.

Informed consent Not applicable; this is a review article. An informed consent statement is provided in each of the studies reviewed.

Open Access This article is distributed under the terms of the Creative Commons Attribution 4.0 International License (http:// creativecommons.org/licenses/by/4.0/), which permits unrestricted use, distribution, and reproduction in any medium, provided you give appropriate credit to the original author(s) and the source, provide a link to the Creative Commons license, and indicate if changes were made.

\section{References}

1. Nobili F, Arbizu J, Bouwman F, Drzezga A, Filippi M, Nestor P, et al. EAN-EANM recommendations for the use of brain $18 \mathrm{~F}-$ Fluorodeoxyglucose Positron Emission Tomography (FDG-PET) in neurodegenerative cognitive impairment and dementia: Delphi consensus. Eur J Neurol. 2018.

2. Jellinger KA. Dementia with Lewy bodies and Parkinson's diseasedementia: current concepts and controversies. J Neural Transm. 2018;125(4):615-50. https://doi.org/10.1007/s00702-017-1821-9.

3. Schrag A, Siddiqui UF, Anastasiou Z, Weintraub D, Schott JM. Clinical variables and biomarkers in prediction of cognitive impairment in patients with newly diagnosed Parkinson's disease: a cohort study. Lancet Neurol. 2017;16(1):66-75. https://doi.org/10.1016/ S1474-4422(16)30328-3.

4. Kovacs GG. Invited review: neuropathology of tauopathies: principles and practice. Neuropathol Appl Neurobiol. 2015;41:3-23. https://doi.org/10.1111/nan.12208.

5. Höglinger GU, Respondek G, Stamelou M, Kurz C, Josephs KA, Lang AE, et al. Clinical diagnosis of progressive supranuclear palsy: the Movement Disorder Society criteria. Mov Disord. 2017;32: 853-64. https://doi.org/10.1002/mds.26987.

6. Boccardi M, Festari C, Altomare D, Gandolfo F, Orini S, Nobili F, et al. Assessing accuracy diagnostic FDG-PET studies to define clinical use for dementia diagnosis. Eur J Nucl Med Mol Imaging. 2018. https://doi.org/10.1007/s00259-018-4024-1.

7. Leone MA, Brainin M, Boon P, Pugliatti M, Keindl M, Bassetti CL. Guidance for the preparation of neurological management guidelines by EFNS scientific task forces - revised recommendations 2012. Eur J Neurol. 2013;20:410-9. https://doi.org/10.1111/ene.12043.

8. Moher D, Liberati A, Tetzlaff J, Altman DG. Preferred reporting items for systematic reviews and meta-analyses: the PRISMA statement. J Clin Epidemiol. 2009;62:1006-12. https://doi.org/10.1016/ j.jclinepi.2009.06.005.

9. Drzezga A, Altomare D, Festari C, Arbizu J, Orini S, Herholz K, et al. Clinical utility of FDG-PET in the evaluation of conditions at risk for AD. Eur J Nucl Med Mol Imaging. 2018. https://doi.org/10. 1007/s00259-018-4032-1.

10. Agosta F, Altomare D, Festari C, Orini S, Gandolfo F, Boccardi M, et al. Clinical utility of FDG-PET in amyotrophic lateral sclerosis 
and Huntington's disease. Eur J Nucl Med Mol Imaging. 2018. https://doi.org/10.1007/s00259-018-4033-0.

11. Edison P, Ahmed I, Fan Z, Hinz R, Gelosa G, Ray Chaudhuri K, et al. Microglia, amyloid, and glucose metabolism in Parkinson's disease with and without dementia. Neuropsychopharmacology. 2013;38:938-49. https://doi.org/10.1038/npp.2012.255.

12. Hosokai Y, Nishio Y, Hirayama K, Takeda A, Ishioka T, Sawada Y, et al. Distinct patterns of regional cerebral glucose metabolism in Parkinson's disease with and without mild cognitive impairment. Mov Disord. 2009;24:854-62. https://doi.org/10.1002/mds.22444.

13. Jokinen P, Scheinin N, Aalto S, Någren K, Savisto N, Parkkola R, et al. [11C]PIB-, [18F]FDG-PET and MRI imaging in patients with Parkinson's disease with and without dementia. Parkinsonism Relat Disord. 2010;16:666-70. https://doi.org/10.1016/j.parkreldis.2010.08. 021.

14. Peppard RF, Martin WR, Carr GD, Grochowski E, Schulzer M, Guttman M, et al. Cerebral glucose metabolism in Parkinson's disease with and without dementia. Arch Neurol. 1992;49:1262-8.

15. Perneczky R, Drzezga A, Boecker H, Ceballos-Baumann AO, Granert O, Förstl H, et al. Activities of daily living, cerebral glucose metabolism, and cognitive reserve in Lewy body and Parkinson's disease. Dement Geriatr Cogn Disord. 2008;26:475-81. https://doi. org/10.1159/000167791.

16. Sasaki M, Ichiya Y, Hosokawa S, Otsuka M, Kuwabara Y, Fukumura T, et al. Regional cerebral glucose metabolism in patients with Parkinson's disease with or without dementia. Ann Nucl Med. 1992;6:241-6.

17. Wu JC, Iacono R, Ayman M, Salmon E, Lin SD, Carlson J, et al. Correlation of intellectual impairment in Parkinson's disease with FDG PET scan. Neuroreport. 2000;11:2139-44.

18. Eberling JL, Richardson BC, Reed BR, Wolfe N, Jagust WJ. Cortical glucose metabolism in Parkinson's disease without dementia. Neurobiol Aging. 1994;15:329-35.

19. Nagano-Saito A, Kato T, Arahata Y, Washimi Y, Nakamura A, Abe $\mathrm{Y}$, et al. Cognitive- and motor-related regions in Parkinson's disease: FDOPA and FDG PET studies. Neuroimage. 2004;22:55361. https://doi.org/10.1016/j.neuroimage.2004.01.030.

20. Griffith HR, den Hollander JA, Okonkwo OC, O'Brien T, Watts RL, Marson DC. Brain metabolism differs in Alzheimer's disease and Parkinson's disease dementia. Alzheimers Dement. 2008;4: 421-7. https://doi.org/10.1016/j.jalz.2008.04.008.

21. Gasca-Salas C, Clavero P, García-García D, Obeso JA, RodríguezOroz MC. Significance of visual hallucinations and cerebral hypometabolism in the risk of dementia in Parkinson's disease patients with mild cognitive impairment. Hum Brain Mapp. 2016;37: 968-77. https://doi.org/10.1002/hbm.23080.

22. Yong SW, Yoon JK, An YS, Lee PH. A comparison of cerebral glucose metabolism in Parkinson's disease, Parkinson's disease dementia and dementia with Lewy bodies. Eur J Neurol. 2007;14: 1357-62. https://doi.org/10.1111/j.1468-1331.2007.01977.x.

23. Bohnen NI, Koeppe RA, Minoshima S, Giordani B, Albin RL, Frey $\mathrm{KA}$, et al. Cerebral glucose metabolic features of Parkinson disease and incident dementia: longitudinal study. J Nucl Med. 2011;52: 848-55. https://doi.org/10.2967/jnumed.111.089946.

24. Tard C, Demailly F, Delval A, Semah F, Defebvre L, Dujardin K, et al. Hypometabolism in posterior and temporal areas of the brain is associated with cognitive decline in Parkinson's disease. J Parkinsons Dis. 2015;5:569-74. https://doi.org/10.3233/JPD-150583.

25. Huang C, Mattis P, Perrine K, Brown N, Dhawan D, Eidelberg D. Metabolic abnormalities associated with mild cognitive impairment in Parkinson disease. Neurology. 2008;70:1470-7.

26. Huang C, Mattis P, Tang C, Perrine K, Carbon M, Eidelberg D. Metabolic brain networks associated with cognitive function in Parkinson's disease. Neuroimage. 2007;34:714-23. https://doi. org/10.1016/j.neuroimage.2006.09.003.
27. Meles SK, Tang CC, Teune LK, Dierckx RA, Dhawan V, Mattis PJ, et al. Abnormal metabolic pattern associated with cognitive impairment in Parkinson's disease: a validation study. J Cereb Blood Flow Metab. 2015;35:1478-84. https://doi.org/10.1038/jcbfm.2015.112.

28. Garibotto V, Montandon ML, Viaud CT, Allaoua M, Assal F, Burkhard PR, et al. Regions of interest-based discriminant analysis of DaTSCAN SPECT and FDG-PET for the classification of dementia. Clin Nucl Med. 2013;38:e112-7. https://doi.org/10.1097/ RLU.0b013e318279b991.

29. Amtage F, Hellwig S, Kreft A, Spehl T, Glauche V, Winkler C, et al. Neuronal correlates of clinical asymmetry in progressive supranuclear palsy. Clin Nucl Med. 2014;39:319-25. https://doi. org/10.1097/RLU.0000000000000373.

30. Amtage F, Maurer C, Hellwig S, Tüscher O, Kreft A, Weiller C, et al. Functional correlates of vertical gaze palsy and other ocular motor deficits in PSP: an FDG-PET study. Parkinsonism Relat Disord. 2014;20:898-906. https://doi.org/10.1016/j.parkreldis.2014.05.013.

31. Blin J, Horwitz B, Baron JC, Agid Y. Does frontal cortex hypometabolism in progressive supranuclear palsy result from subcortical dysfunction? Eur J Neurol. 1995;1:221-8. https://doi.org/ 10.1111/j.1468-1331.1995.tb00075.x.

32. Botha H, Whitwell JL, Madhaven A, Senjem ML, Lowe V, Josephs KA. The pimple sign of progressive supranuclear palsy syndrome. Parkinsonism Relat Disord. 2014;20:180-5. https://doi.org/10. 1016/j.parkreldis.2013.10.023.

33. Eckert T, Tang C, Ma Y, Brown N, Lin T, Frucht S, et al. Abnormal metabolic networks in atypical parkinsonism. Mov Disord. 2008;23:727-33. https://doi.org/10.1002/mds.21933.

34. Foster NL, Gilman S, Berent S, Sima AA, D'Amato C, Koeppe RA, et al. Progressive subcortical gliosis and progressive supranuclear palsy can have similar clinical and PET abnormalities. J Neurol Neurosurg Psychiatry. 1992;55:707-13.

35. Garraux G, Salmon E, Degueldre C, Lemaire C, Franck G. Medial temporal lobe metabolic impairment in dementia associated with motor neuron disease. J Neurol Sci. 1999;168:145-50. https://doi. org/10.1016/S0022-510X(99)00188-4.

36. Hoffman JM, Welsh-Bohmer KA, Hanson M, Crain B, Hulette $\mathrm{CM}$, Earl N, et al. FDG PET imaging in patients with pathologically verified dementia. J Nucl Med. 2000;41:1920-8.

37. Hosaka K, Ishii K, Sakamoto S, Mori T, Sasaki M, Hirono N, et al. Voxel-based comparison of regional cerebral glucose metabolism between PSP and corticobasal degeneration. J Neurol Sci. 2002;199:67-71.

38. Juh R, Kim J, Moon D, Choe B, Suh T. Different metabolic patterns analysis of parkinsonism on the 18F-FDG PET. Eur J Radiol. 2004;51:223-33. https://doi.org/10.1016/S0720048X(03)00214-6.

39. Karbe H, Grond M, Huber M, Herholz K, Kessler J, Heiss WD. Subcortical damage and cortical dysfunction in progressive supranuclear palsy demonstrated by positron emission tomography. J Neurol. 1992;239:98-102. https://doi.org/10.1007/BF00862982.

40. Nagahama Y, Fukuyama H, Turjanski N, Kennedy A, Yamauchi H, Ouchi Y, et al. Cerebral glucose metabolism in corticobasal degeneration: comparison with progressive supranuclear palsy and normal controls. Mov Disord. 1997;12:691-6. https://doi.org/10.1002/ mds.870120510.

41. Niethammer M, Tang CC, Feigin A, Allen PJ, Heinen L, Hellwig S, et al. A disease-specific metabolic brain network associated with corticobasal degeneration. Brain. 2014;137:3036-46. https://doi. org/10.1093/brain/awu256.

42. Peigneux P, Salmon E, Garraux G, Laureys S, Willems S, Dujardin $\mathrm{K}$, et al. Neural and cognitive bases of upper limb apraxia in corticobasal degeneration. Neurology. 2001;57:1259-68. https:// doi.org/10.1212/WNL.57.7.1259.

43. Piccini P, de Yebenez J, Lees AJ, Ceravolo R, Turjanski N, Pramstaller P, et al. Familial progressive supranuclear palsy: 
detection of subclinical cases using 18F-dopa and 18 fluorodeoxyglucose positron emission tomography. Arch Neurol. 2001;58:1846-51.

44. Takahashi R, Ishii K, Kakigi T, Yokoyama K, Mori E, Murakami T. Brain alterations and Mini-Mental State Examination in patients with progressive supranuclear palsy: voxel-based investigations using F-fluorodeoxyglucose positron emission tomography and magnetic resonance imaging. Dement Geriatr Cogn Disord Extra. 2011;1:381-92. https://doi.org/10.1159/000333368.

45. Zalewski N, Botha H, Whitwell JL, Lowe V, Dickson DW, Josephs KA. FDG-PET in pathologically confirmed spontaneous 4Rtauopathy variants. J Neurol. 2014;261:710-6. https://doi.org/10. 1007/s00415-014-7256-4.

46. Zwergal A, La Fougère C, Lorenzl S, Rominger A, Xiong G, Deutschenbaur L, et al. Postural imbalance and falls in PSP correlate with functional pathology of the thalamus. Neurology. 2011;77: 101-9. https://doi.org/10.1212/WNL.0b013e318223c79d.

47. Zwergal A, La Fougère C, Lorenzl S, Rominger A, Xiong G, Deutschenbaur L, et al. Functional disturbance of the locomotor network in progressive supranuclear palsy. Neurology. 2013;80: 634-41. https://doi.org/10.1212/WNL.0b013e318281cc43.

48. Eckert T, Barnes A, Dhawan V, Frucht S, Gordon MF, Feigin AS, et al. FDG PET in the differential diagnosis of parkinsonian disorders. Neuroimage. 2005;26:912-21. https://doi.org/10.1016/j. neuroimage.2005.03.012.

49. Garraux G, Phillips C, Schrouff J, Kreisler A, Lemaire C, Degueldre C, et al. Multiclass classification of FDG PET scans for the distinction between Parkinson's disease and atypical parkinsonian syndromes. Neuroimage Clin. 2013;2:883-93. https://doi. org/10.1016/j.nicl.2013.06.004.

50. Hellwig S, Reinhard M, Amtage F, Guschlbauer B, Buchert R, Tüscher O, et al. Transcranial sonography and $[18 \mathrm{~F}]$ fluorodeoxyglucose positron emission tomography for the differential diagnosis of parkinsonism: a head-to-head comparison. Eur J Neurol. 2014;21:860-6. https://doi.org/10.1111/ene.12394.

51. Tripathi M, Dhawan V, Peng S, Kushwaha S, Batla A, Jaimini A, et al. Differential diagnosis of parkinsonian syndromes using F-18 fluorodeoxyglucose positron emission tomography. Neuroradiology. 2013;55:483-92. https://doi.org/10.1007/s00234-012-1132-7.

52. Akdemir ÜÖ, Tokçaer AB, Karakuș A, Kapucu LÖ. Brain 18FFDG PET imaging in the differential diagnosis of parkinsonism. Clin Nucl Med. 2014;39:e220-6. https://doi.org/10.1097/RLU. 0000000000000315 .

53. Derlin T, Afzal W, Wilke F, Apostolova I, Klutmann S, Meyer PT, et al. IBZM SPECT and FDG PET in the differential diagnosis of parkinsonian syndromes: comparison with respect to inter-rater agreement. Nuklearmedizin. 2010;49:139-47. https://doi.org/10. 3413/nukmed-0290.

54. Hellwig S, Frings L, Amtage F, Buchert R, Spehl TS, Rijntjes M, et al. 18F-FDG PET is an early predictor of overall survival in suspected atypical parkinsonism. J Nucl Med. 2015;56:1541-6. https://doi.org/10.2967/jnumed.115.159822.

55. Klein RC, de Jong BM, de Vries JJ, Leenders KL. Direct comparison between regional cerebral metabolism in progressive supranuclear palsy and Parkinson's disease. Mov Disord. 2005;20:1021-30. https://doi.org/10.1002/mds.20493.

56. Park HK, Kim JS, Im KC, Oh SJ, Kim MJ, Lee JH, et al. Functional brain imaging in pure akinesia with gait freezing: [18F]FDG PET and [18F]FP-CIT PET analyses. Mov Disord. 2009;30:237-45. https://doi.org/10.1002/mds.22347.

57. Teune LK, Bartels AL, De Jong BM, Willemsen ATM, Eshuis SA, De Vries JJ, et al. Typical cerebral metabolic patterns in neurodegenerative brain diseases. Mov Disord. 2010;25:2395-404. https:// doi.org/10.1002/mds.23291.

58. Zhao P, Zhang B, Gao S. 18[F]-FDG PET study on the idiopathic Parkinson's disease from several parkinsonian-plus syndromes.
Parkinsonism Relat Disord. 2012;18:S60-2. https://doi.org/10. 1016/S1353-8020(11)70020-7.

59. Mudali D, Teune LK, Renken RJ, Leenders KL, Roerdink JB. Classification of parkinsonian syndromes from FDG-PET brain data using decision trees with SSM/PCA features. Comput Math Methods Med. 2015;2015:136921. https://doi.org/10.1155/2015/136921.

60. Srulijes K, Reimold M, Liscic RM, Bauer S, Dietzel E, LiepeltScarfone I, et al. Fluorodeoxyglucose positron emission tomography in Richardson's syndrome and progressive supranuclear palsyparkinsonism. Mov Disord. 2012;27:151-5.

61. Hellwig S, Amtage F, Kreft A, Buchert R, Winz OH, Vach W, et al. [18F]FDG-PET is superior to [123I]IBZM-SPECT for the differential diagnosis of parkinsonism. Neurology. 2012;79:1314-22. https://doi.org/10.1212/WNL.0b013e31826c1b0a.

62. Tang CC, Poston KL, Eckert T, Feigin A, Frucht S, Gudesblatt M, et al. Differential diagnosis of parkinsonism: a metabolic imaging study using pattern analysis. Lancet Neurol. 2010;9:149-58. https:// doi.org/10.1016/S1474-4422(10)70002-8.

63. Tripathi M, Tang CC, Feigin A, De Lucia I, Nazem A, Dhawan V, et al. Automated differential diagnosis of early parkinsonism using metabolic brain networks: a validation study. J Nucl Med. 2016;57: 60-6. https://doi.org/10.2967/jnumed.115.161992.

64. Sha SJ, Ghosh PM, Lee SE, Corbetta-Rastelli C, Jagust WJ, Kornak J, et al. Predicting amyloid status in corticobasal syndrome using modified clinical criteria, magnetic resonance imaging and fluorodeoxyglucose positron emission tomography. Alzheimers Res Ther. 2015;7:8. https://doi.org/10.1186/ s13195-014-0093-y.

65. Taswell C, Villemagne VL, Yates P, Shimada H, Leyton CE, Ballard KJ, et al. 18F-FDG PET improves diagnosis in patients with focal-onset dementias. J Nucl Med. 2015;56:1547-53. https://doi.org/10.2967/jnumed.115.161067.

66. Cordato NJ, Halliday GM, McCann H, Davies L, Williamson P, Fulham M, et al. Corticobasal syndrome with tau pathology. Mov Disord. 2001;16:656-67. https://doi.org/10.1002/mds.1124.

67. Pilotto A, Premi E, Paola Caminiti S, Presotto L, Turrone R, Alberici A, et al. Single-subject SPM FDG-PET patterns predict risk of dementia progression in Parkinson disease. Neurology. 2018;90(12):e1029-37. https://doi.org/10.1212/WNL.0000000000005161.

68. Kimura N, Hanaki S, Masuda T, Hanaoka T, Hazama Y, Okazaki T, et al. Brain perfusion differences in parkinsonian disorders. Mov Disord. 2011;26:2530-7. https://doi.org/10.1002/mds.23915.

69. Varrone A, Pagani M, Salvatore E, Salmaso D, Sansone V, Amboni $\mathrm{M}$, et al. Identification by [99mTc]ECD SPECT of anterior cingulate hypoperfusion in progressive supranuclear palsy, in comparison with Parkinson's disease. Eur J Nucl Med Mol Imaging. 2007;34: 1071-81. https://doi.org/10.1007/s00259-006-0344-7.

70. Nobili F, Festari C, Altomare D, Agosta F, Orini S, Van Laere K, et al. Automated assessment of FDG-PET for the differential diagnosis in patients with neurodegenerative disorders. Eur J Nucl Med Mol Imaging. 2018. https://doi.org/10.1007/s00259-018-4030-3.

71. Niethammer M, Feigin A, Eidelberg D. Functional neuroimaging in Parkinson's disease. Cold Spring Harb Perspect Med. 2012;2: a009274. https://doi.org/10.1101/cshperspect.a009274.

72. Varrone A, Asenbaum S, Vander Borght T, Booij J, Nobili F, Någren K, et al. EANM procedure guidelines for PET brain imaging using [18F]FDG, version 2. Eur J Nucl Med Mol Imaging. 2009;36:2103-10. https://doi.org/10.1007/s00259-009-1264-0.

73. Whitwell JL, Höglinger GU, Antonini A, Bordelon Y, Boxer AL, Colosimo C, et al. Radiological biomarkers for diagnosis in PSP: where are we and where do we need to be? Mov Disord. 2017;32: 955-71. https://doi.org/10.1002/mds.27038.

74. Poston KL, Eidelberg D. FDG PET in the evaluation of Parkinson's disease. PET Clin. 2010;5:55-64. https://doi.org/10.1016/j.cpet. 2009.12.004. 